\title{
DUAL CHANNEL 115 AND 230 GHZ SIS RECEIVERS IN OPERATION AT THE OWENS VALLEY RADIO OBSERVATORY
}

\author{
D. P. Woody and C. J. Giovanine \\ Owens Valley Radio Observatory \\ California Institute of Technology \\ Big Pine, CA 93513
}

R. E. Miller

AT\&T Bell Labs

600 Mountain Avenue

Murray Hill, NJ 07974

\section{Abstract}

The Owens Valley Radio Observatory millimeter-wave interferometer array is presently operating with dual channel SIS tunnel junction receivers. The first channel covers the frequency range from 85 to $120 \mathrm{GHz}$ and the second channel covers the frequency range from 200 to $300 \mathrm{GHz}$. The mixers consist of a corrugated feedhorn, single-stage circular to rectangular waveguide transition, reduced-height waveguide with an SIS junction mounted across the E-plane and a non-contacting backshort. The mixer block has a built-in RF choke for the IF signal path which is designed to present a short circuit to the junction at frequencies above the $2 \mathrm{GHz}$ IF frequency. The small area $\left(<1 \mu \mathrm{m}^{2}\right)$ PbInAu-native oxide-PbAu SIS tunnel junctions are fabricated using a bridge lift-off technique. The LO power is provided by Gunn oscillators followed by doublers or triplers. The receivers in the 85 to $120 \mathrm{GHz}$ band have noise temperatures of $<100 \mathrm{~K}$, while the receivers in the 200 to $300 \mathrm{GHz}$ band have noise temperatures in the range from 200 to $300 \mathrm{~K}$. These dual channel receivers are mounted in $4.5 \mathrm{~K}$ closed cycle refrigerators. They are in continuous use on the three element millimeter-wavelength interferometer array.

\section{Introduction}

Astronomical observations at millimeter and submillimeter frequencies are often limited by the receiver sensitivity. It is important to use the lowest noise receivers available without compromising system reliability. This is especially true for radio interferometer arrays where the loss or degradation of any one of the many receivers seriously compromises the quality of the data. The theory of mixing in SIS tunnel junctions predicts that their noise temperature should approach the quantum limit of hv/k throughout the millimeter and submillimeter frequency range 1,2,3. And indeed SIS mixers have produced the lowest noise receivers in this frequency range $2,4,5,6$, although they have not yet reached the quantum limit.

There has been some concern that these devices are not reliable enough for use on systems operating continuously and there are several areas where SIS junctions might suffer reliability problems. These include storage lifetime, susceptibility to electrical transients, and survival of thermal cycling. The reliability of the cryogenic cooling system is also important. We have used SIS receivers exclusively in the millimeter array since 1980 and our experience with the devices shows that with proper design and a few simple precautions they perform very reliably.

In this paper we describe the dual channel SIS receivers which are currently being used in the OVRO millimeter array. The receivers are an improved and augmented version of the original system7. The main changes have been the addition of the 200 to $300 \mathrm{GHz}$ channel, switching from circular waveguide mixer blocks and backshorts to reduced-height rectangular waveguide, and the use of closed cycle $4.5 \mathrm{~K}$ refrigerators of our own design and construction.

\section{Receiver system}

The OVRO millimeter array consists of three 10.4 meter diameter telescopes, receivers, and the associated electronics for performing aperture synthesis mapping of astronomical objects at wavelengths from one to three millimeters8. The telescopes use a Nasmyth optical system which brings the signal beam into a sidecab attached to the telescope mount. This provides a convenient place for the receiver system which is out of the weather and easily accessible.

A schematic drawing of one channel of the receiver system is shown in Figure 1. The major components are the local oscillator source for the heterodyne mixer, the mixer block with its feedhorn and backshort, the IF amplifier, and the cryogenic system for cooling the mixer. As many as four different receivers can be mounted in the same cryostat. The array is currently operating with two receivers in each cryostat.

An important advantage of SIS receivers is that they require less than a microwatt of LO power. This is obtained by multiplying the output from low power solid state oscillators. The LO for the 85 to $120 \mathrm{GHz}$ band is obtained by doubling the outpu from a Gunn oscillator in the 42 to $60 \mathrm{GHz}$ range. For the 200 to $300 \mathrm{GHz}$ band the LO is obtained by tripling the output from a Gunn oscillator in the 66 to $100 \mathrm{GHz}$ range. A thin mylar beamsplitter is used to reflect $-1 \%$ of the multiplier output into the mixer feedhorn while $-99 \%$ of the signal is transmitted through the beam-splitter.

The mixers are cooled to $4.5 \mathrm{~K}$ in a closed cycle refrigerator system. This system uses a Gifford-McMahon displacement cooler manufactured by CTI to reach $12 \mathrm{~K}$ with an intermediate stage at $50 \mathrm{~K}$. The radiation shields are attached to the $50 \mathrm{~K}$ stage. A JT (Joule-Thompson) loop is used to reach $4.5 \mathrm{~K}$ The compressor for the cryogenic system is produced by Cryosystems and provides the helium gas at $300 \mathrm{psi}$ with returns a $60 \mathrm{psi}$ from the Gifford-McMahon cooler and $16 \mathrm{psi}$ from the JT loop.

The heat exchangers for the JT loop are of a simple design consisting of a pair of $5 \mathrm{~mm}$ diameter copper refrigeration tubes inside a $2 \mathrm{~m}$ length of $13 \mathrm{~mm}$ diameter copper refrigeration tubing. The two $5 \mathrm{~mm}$ diameter tubes provide redundant high pressure input lines. The low pressure return path is in the space between the outer tube and the two smaller tubes. The outer tube is squashed after the smaller tubes have been inserted to improve the thermal conductance between the two counter-flowing gas streams. The $2 \mathrm{~m}$ long assembly is then wound in a spiral to fit into the cryostat. The use of redundant high pressure input lines gives a spare which can be used in the event of a plug in one tube Surprisingly most of the plugs appear in the high pressure lines and not at the JT orifice. Under normal operating conditions a plug will form after $\sim 8$ weeks of operation. Recovery from a JT plug is not a serious interruption since the cycle time for the system from cold to warm to cold again is less than 12 hours.

Manuscript received August 22, 1988.

0018-9464/89/0300-1366\$01.00@1989 IEEE 


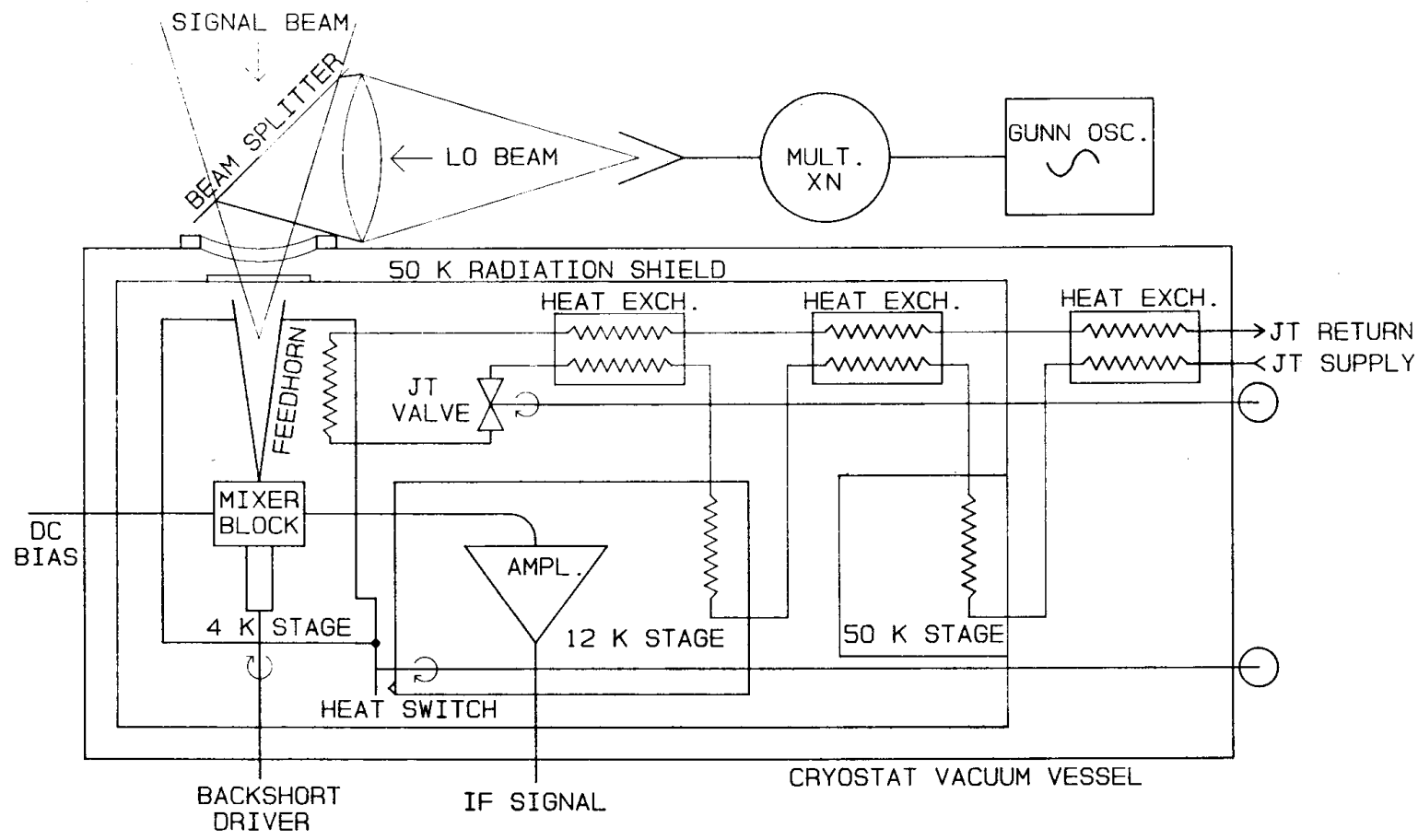

Figure 1. Schematic drawing of a single channel of the receiver.

Mixer blocks

The JT valve has a variable orifice so that the flow rate can be varied to optimize the trade-offs between cooling capacity and ultimate temperature. The JT loop has a cooling capacity of $\sim 1$ Watt at $4.5 \mathrm{~K}$ using a return pressure of $16 \mathrm{psi}$. The cooling capacity is -.3 Watts at $3 \mathrm{~K}$ when a vacuum pump is used in series with the compressor on the JT low pressure return line.

The cryostat is a $25 \mathrm{~cm} \times 25 \mathrm{~cm} \times 60 \mathrm{~cm}$ rectangular box. It is constructed from welded $1 \mathrm{~cm}$ thick aluminum plates. The access for working inside the dewar is through a pair of large 20 $\mathrm{cm} \times 56 \mathrm{~cm}$ rectangular openings. One of the openings faces directly into the sidecab room which gives convenient access to the inside of the cryostat while the receiver is mounted on the telescope. This makes installing and servicing of the receiver quit easy. Half of the volume is occupied by the closed cycle refrigerator system which leaves a $25 \mathrm{~cm} \times 25 \mathrm{~cm} \times 25 \mathrm{~cm}$ cube for the receiver system. The mounting surfaces and radiation shield are supported on G-10 fiber-glass epoxy standoffs attached to the vacuum shroud close to the optical windows. The mixer blocks and feedhorns move less than $.1 \mathrm{~mm}$ relative to the optical windows when cooled from room temperature to $4.5 \mathrm{~K}$ and are mechanically isolated from the $-1 \mathrm{sec}$ pulses from the Gifford-McMahon cooler. This is important for an interferometer which is sensitive to receiver motions of $\sim 1 \%$ of a wavelength.

The vacuum windows in the cryostat are made of $25 \mu \mathrm{m}$ thick mylar film. Double windows with an air gap between them are used to avoid fogging on humid days. A half-wavelength thick crystalline quartz window with a black polyethylene coating is mounted on the $50 \mathrm{~K}$ radiation shield to block infrared radiation. Another layer of black polyethylene is mounted across the input of the cooled feedhorn to further reduce the infrared loading on the SIS junction.

The IF amplifiers are copies of the 1.2 to $1.7 \mathrm{GHz}$ GaAs FET amplifiers developed by NRAO9. They are mounted on the 12 $\mathrm{K}$ stage of the Gifford-McMahon cooler and have noise temperatures of 10 to $15 \mathrm{~K}$ over the $500 \mathrm{MHz}$ bandwidth.
The mixer blocks in the receiver system are of a fairly straightforward design. The mixer assembly consists of a feedhorn, a transition plate, the mixer block and an adjustable backshort. The feedhorn is a corrugated feed which has a Gaussian beam pattern with an opening angle of 30 degrees. A single half wavelength transition section is used to match the circular waveguide from the feedhorn to the reduced-height rectangular waveguide of the mixer block containing the junction and backshort. This simple transition gives better than $-20 \mathrm{~dB}$ input return loss over $\sim 16 \%$ bandwidth and better than $-10 \mathrm{~dB}$ over half an octave.

The mixer block contains the SIS tunnel junction and part of the IF and bias circuitry. An isometric view of the central section of the mixer block looking from the feedhorn and transition plate towards the backshort is shown in Figure 2. The waveguide for the 85 to $120 \mathrm{GHz}$ blocks is $2.0 \mathrm{~mm} \times .25 \mathrm{~mm}$ and that for the 200 to $300 \mathrm{GHz}$ blocks is $1.0 \mathrm{~mm} \times .25 \mathrm{~mm}$.

An important feature of the mixer design is that the RF choke structure is built into the block and is not a part of the junction chip. Although many mixers have been built this way, it is now common practice to put the RF choke onto the chip containing the junction. The purpose of the choke is to short the RF currents to the waveguide wall while coupling the IF signal and DC bias to the outside. The choke has a coaxial structure. The inner conductor has a uniform square cross section along its length while the $\lambda / 4$ sections of low and high impedance transmission line are generated by varying the cross section of the outer conductor formed by the copper mixer block itself. The transition plate covers the top of the RF choke seen in figure 2 and forms part of the outer conductor. The inner square conductor is insulated from the block by $6 \mu \mathrm{m}$ thick mylar. The high impedance sections are $55 \Omega$. The low impedance sections are only $1.5 \Omega$. This is a much lower impedance than can be easily achieved using standard stripline techniques and also produces a much larger ratio of high to low impedances. It takes only a three section choke to effectively terminate the RF currents in a perfect short at the waveguide wall. 


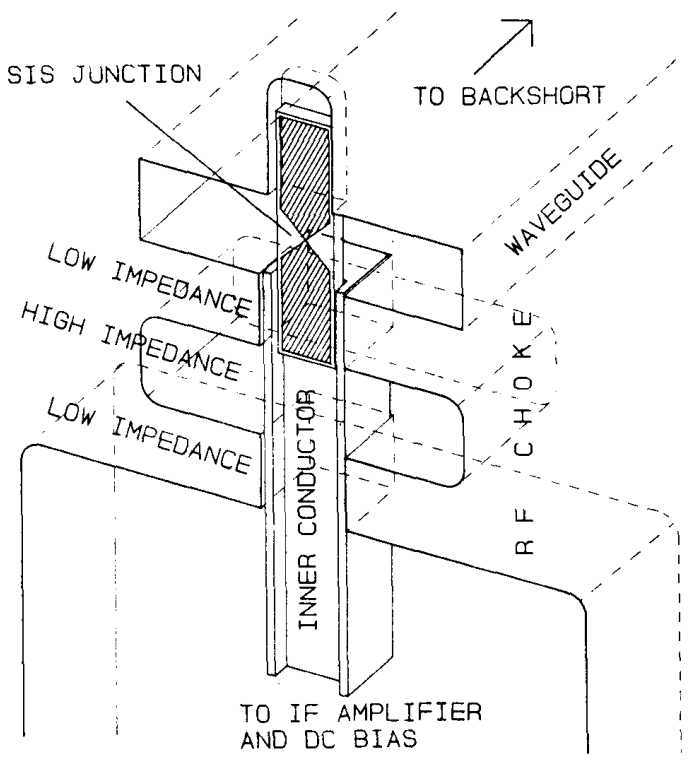

Figure 2. Isometric view of the mixer block as seen from the direction of the incoming radiation. Only the hidden lines for the pockets and waveguide machined into the copper block are shown.

There are recessed slots in the inner conductor of the choke and on the opposite wall of the waveguide for mounting the $1 \mathrm{~mm}$ $x .3 \mathrm{~mm} \times .1 \mathrm{~mm}$ chip containing the junction. Silver paint is used to glue the chip in the slot film side down with the junction centered in the waveguide. This technique minimizes the thickness of paint through which the RF currents must pass. Since the tuning structures are built into the block and the contact technique requires no special soldering or film preparation, a wide variety of junctions can be used. We use the same junction chips for both receiver channels since there is no tuned RF circuitry on the chips.

There are also electrical advantages to fabricating the choke structure in the mixer block. SIS mixers are prone to saturation at modest output power levels 10,11 . This is because they achieve their optimum performance over a bias voltage range much less than the photon assisted tunneling step size of hv/e. Thus it is important to terminate unwanted signals in electrical shorts as seen by the junction. Figure 3 is an electrical schematic of the IF and DC circuit. The low impedance sections of the RF choke are essentially lumped capacitors at the IF frequency and serve as the first element in a pi-network lowpass filter. This circuit transforms the IF impedance of the LO biased junction from $-100 \Omega$ down to $50 \Omega$ to match to the IF amplifier ${ }^{12}$. Frequencies above the $2 \mathrm{GHz}$ IF band and up to the RF frequency are terminated in shorts as seen by the junction. This is important for our mixers which have an RF bandwidth greater than $10 \mathrm{GHz}$. Note that because the junctions are efficient down converters even at IF frequencies of many tens of $\mathrm{GHz}$ it only takes a narrow resonant open circuit in the choke structure at almost any frequency less than the RF frequency to give saturation problems.

The SIS tunnel junctions are less then $1 \mu \mathrm{m}^{2}$ and it only takes a very small electrical pulse to blow them up. This has been a problem for many people working with these devices. The simple procedures we use in handling the junctions have eliminated this problem. The junctions are never be connected to "flying" leads even if they are only a few centimeters long. We always use twisted pairs of wires or coaxial lines. For actual operation we put all of the necessary circuit protection in the mixer block itself. All DC lines pass through a standard RFI filter consisting of a coaxial capacitor and series inductor and then pass through a $10 \mathrm{k} \Omega$

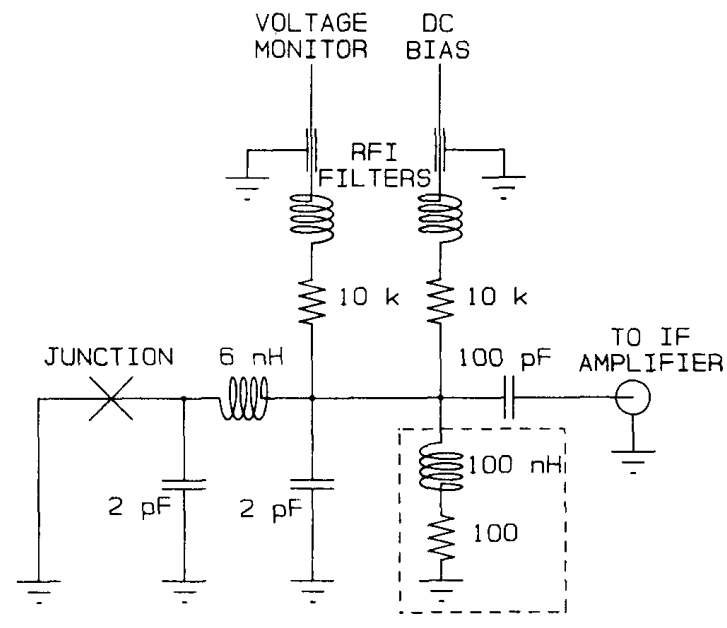

Figure 3. Schematic diagram of IF and DC circuit contained in the mixer block.

resistor before connecting to the junction circuit. The only other connection to the outside world is the IF line which is coupled through a $100 \mathrm{pF}$ capacitor. The DC leads are run out of the cryostat as a twisted bundle to the bias circuit without any further RFI filtering. This has proved to be quite satisfactory and we have never lost a junction during operation even when lightening strikes have destroyed the computers and other electronic equipment in the sidecab.

SIS tunnel junctions can have very high and even negative output impedances. We find that this is only a problem in the 200 to $300 \mathrm{GHz}$ mixers. The shunt shown in the dotted box in Figure 3 is used to present a $100 \Omega \mathrm{DC}$ source impedance to the junctions in these mixers so that the $\mathrm{DC}$ bias will be stable.

A three-stage noncontacting backshort is used. It is connected to a 2-56 screw and nut drive system which is bolted to the mixer block. The drive is connected to the outside of the cryostat via a rotary feedthrough to a computer-controlled stepper motor. We have seen no wear or tuning irregularities associated with the backshorts over the eight years they have been in use.

\section{Junctions}

SIS tunnel junctions used for routine astronomical observations must satisfy several stringent requirements. Not only must they have the necessary electrical properties to produce low noise receivers, they must also be robust enough to survive normal handling, long term storage and many thermal cycles.

The mixer blocks use only a single backshort tuner which has only a limited ability to tune out the capacitive reactance of the SIS sandwich structure. Thus it is necessary to fabricate tunnel junctions with a small $\omega \mathrm{RC}$ product at the operating frequency, i.e. a high current density. The characteristic impedance our waveguide structures is in the range of 50 to $100 \Omega$, which in combination with the high current density requirement implies that small area single junctions or series arrays of junctions must be used.

We have developed a process which satisfies these requirements using lead-alloy electrodes and a variation of the trilevel stencil technique developed by Dunkleberger and Dolan $7,13,14$. This technique allows us to fabricate bridges of photoresist as narrow as one micron and many microns long which are suspended above the substrate. The bridges are used as shadow 


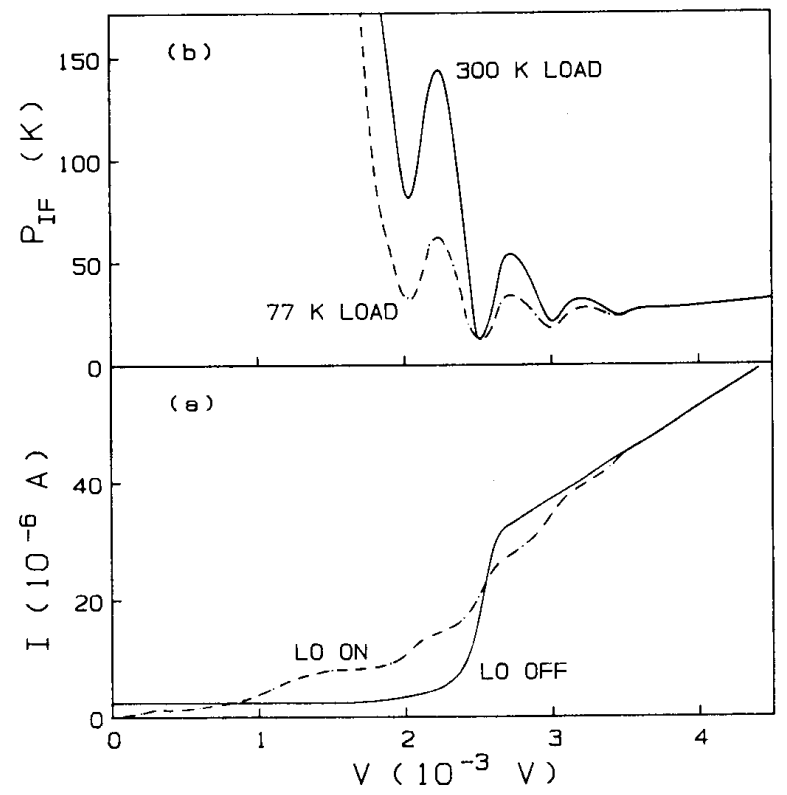

Figure 4. (a) SIS tunnel junction I vs. V curve without (solid) and with (dashed) $112 \mathrm{GHz}$ LO applied. (b) down-converted power coupled to the IF amplifier in the band from 1.2 to $1.7 \mathrm{GHz}$ for 77 and $300 \mathrm{~K}$ loads vs. DC bias voltage.

masks with the top and bottom electrodes evaporated from different angles to give a small overlap (as small as $.5 \mu \mathrm{m} \times .5 \mu \mathrm{m}$ ) beneath the bridge. Oxidation of the bottom electrode to form the dielectric tunnel barrier is done in situ without breaking the vacuum. The chromium mask used to pattern the bridge structure is made using electron-beam lithography.

Our junctions are fabricated with a $\mathrm{Pb}_{90} \mathrm{In}_{8} \mathrm{Au}_{2}$ base electrode and $\mathrm{Pb}_{96} \mathrm{Au}_{4}$ counter electrode on $0.1 \mathrm{~mm}$ thick polycrystalline quartz substrates. Current densities as large as $10^{4}$ $\mathrm{A} / \mathrm{cm}^{2}$ can be obtained and at $100 \mathrm{GHz} \omega \mathrm{RC}$ is estimated to be less than one. Hence the capacitive reactance is not large at the frequencies of interest and the small area junctions have impedances in the range from 30 to $100 \Omega$ which is a reasonable match to our waveguide structures.

Although there are $\mathrm{Pb}$ alloys which produce sharper I vs. $\mathrm{V}$ curves, the $\mathrm{Pb}_{90} \mathrm{In}_{8} \mathrm{Au}_{2}$ alloy base electrode has proved to be very robust and produces excellent low noise receivers. The I vs. V curve for a typical junction is shown in Figure 4. The photon assisted tunneling steps are clearly visible when the $112 \mathrm{GHz} \mathrm{LO}$ voltage is applied $(\mathrm{hv} / \mathrm{e}=.23 \mathrm{mV}$ at $112 \mathrm{GHz})$. The downconverted power, PIF, coupled into the IF amplifier for $300 \mathrm{~K}$ and $77 \mathrm{~K}$ loads is also shown in Figure 4. The conversion efficiency clearly peaks in the middle of the first step below the gap voltage. The temperature scale in the PIF plot is calibrated using the shot noise from the junction 7,15 biased above $4 \mathrm{mV}$. The rapid noise rise below $1.8 \mathrm{mV}$ is associated with the drop-back voltage and the Josephson effect. It can be suppressed by applying a magnetic field, but we have found that in the frequency range covered by our receivers stable low noise operation can be achieved without applying a magnetic field.

A properly fabricated batch of junctions can be stored for at least four years at room temperature in an ordinary bell jar with the normal state resistance changing by $-20 \%$ per year and with no noticeable degradation in the quality of the I vs. V curve. Junctions can be kept in $\mathrm{LN}_{2}$ or in a household freezer for long term storage. We often use junctions in the receivers which were fabricated several years earlier.
Our junctions usually survive more than ten thermal cycles, and even then most failures are traced to open circuits in the silver paint used for mounting the junctions. They do degrade over a period of a week or so on the lab bench or in a dirty (i.e. pump oil contaminated) dewar. We typically use the same junction for more than a year before some mishandling or power failure results in long exposure to humid or oily air and degradation of receiver performance.

\section{Performance Summary}

Table 1 lists the performance of the receivers on the telescope. The primary measurement made is the response of the receivers to ambient temperature and liquid nitrogen cooled absorbers. The interferometer was used to determine the ratio of the receiver response in the two sidebands. Typically the receivers could be tuned to give equal response in both sidebands or -10 times large response in the signal sideband, although some receivers could be tuned to reject the unwanted sideband by more than a factor of 100 . Thus the SSB (single-sideband) receiver noise temperatures are not simply twice the DSB (double-sideband) noise temperatures. The IF noise temperatures and conversion efficiencies are calculated using the junction as a shot noise source when it is bias well above the gap voltage 7,15 .

Table 1. Receiver performance at $115 \mathrm{GHz}$ and $230 \mathrm{GHz}$.

$\begin{array}{lrr} & 115 \mathrm{GHz} & 230 \mathrm{GHz} \\ \mathrm{T}_{\text {REC,DSB }} & 75-125 \mathrm{~K} & 200-300 \mathrm{~K} \\ \mathrm{~T}_{\mathrm{REC}, S S B} & 100-200 \mathrm{~K} & 250-400 \mathrm{~K} \\ \mathrm{~T}_{\mathrm{IF}} & 10-15 \mathrm{~K} & 10-15 \mathrm{~K} \\ \mathrm{~L}_{\mathrm{DSB}}{ }^{-1} & -4 \text { to }-6 \mathrm{~dB} & -8 \text { to }-10 \mathrm{~dB}\end{array}$

Linearity and saturation problems are diagnosed by using absorbers which fill part of the input signal beam. The response to these loads is monitored while the remaining part of the beam is either blocked by a hot load or a cold load. Saturation problems will show up as decreased response when the blocked part of the beam is looking at a hot load. Tests are also made to make sure that the receiver does not change its DC bias point when it is presented with different temperature loads. And of course the ultimate test is to check that the total interferometer system gives the correct response to well characterized astronomical sources. These tests indicate that the saturation effect for a $300 \mathrm{~K}$ load is always less than $10 \%$ and usually less than $2 \%$.

The response to harmonic frequencies is determined from astronomical observations and is less than $1 \%$ for the $115 \mathrm{GHz}$ receivers. This is despite the low $\omega R C$ product of our junctions which suggests that they might be sensitive to harmonics of the LO. SIS quasiparticle mixers apparently are inefficient at harmonic mixing unless special effort is made to optimize them for this purpose.

Further improvements are planned for the receiver systems. The cryostats are designed to hold a total of four receivers and installation of two more receivers will allow dual polarization capability at each frequency, thus increasing the interferometer sensitivity by $\sqrt{2}$. Operation of the cryostat at $3 \mathrm{~K}$ using a vacuum pump assist on the JT retum and the use of HEMT amplifiers is expected to further improve the performance by approximately a factor of two.

A total of six SIS receivers are currently being used in an instrument which has state-of-the-art noise performance and high reliability. This demonstrates that the excellent performance predicted and achieved in the laboratory for superconductor technology can be transferred to field instruments for use by nonexperts. 


\section{Acknowledgements}

It is a pleasure to acknowledge the support of the staff of the Owens Valley Radio Observatory in the development and construction of the dual channel receivers described in this paper. The OVRO millimeter array is supported by NSF grant AST 87 14405.

\section{References}

[1] J. R. Tucker, "Quantum limited detection in tunnel junction mixers," IEEE J. Quantum Electron., vol. QE-15, pp. 1234 1258, Nov. 1979.

[2] J. R. Tucker and M. J. Feldman, "Quantum detection at millimeter wavelengths," Rev. Modern Phys., vol. 57, pp. 1055-1113, Oct. 1985

[3] M. J. Wengler and D. P. Woody, "Quantum noise in heterodyne detection," IEEE J. Quantum Electron., vol. QE-23, pp. 613-622, May 1987.

[4] T. G. Phillips and D. P. Woody, "Millimeter- and submillimeter-wave receivers," Ann. Rev. Astron. Astrophys., vol. 20, pp. 285-321, 1982.

[5] L. R. D'Addario, "An SIS mixer for $90-120 \mathrm{GHz}$ with gain and wide bandwidth," Int. J. Infrared and Millimeter Waves, vol. 5, pp. 1419-1442, 1984

[6] B. N. Ellison and R. E. Miller, "A low noise $230 \mathrm{GHz}$ SIS receiver," Int. J. of Infrared and Millimeter Waves, vol. 8 pp. $608-625,1987$

[7] D. P. Woody, R. E. Miller, and M. J. Wengler "85-115 $\mathrm{GHz}$ receivers for radio astronomy," IEEE Tran Microwave Theory Tech., vol. MTT-33, pp. 90-95, Feb. 1985
[8] C. R. Masson, et al., "The Caltech millimeter wave interferometer," Union Radio-Scientifique Internationale Symposium on Millimeter and Sub-Millimeter Wave Radio Astronomy, Granada, Spain, pp. 65-74, Sept. 1984.

[9] S. Weinreb, D. L. Fenstermacher, and R. W. Harris, "Ultralow-noise 1.2 to $1.7 \mathrm{GHz}$ cooled GaAs-FET amplifiers," IEEE Trans. Microwave Theory Tech., vol. MTT-30, pp. 849-853, June, 1982.

[10] A. D. Smith and P. L. Richards, "Analytical solutions to superconductor-insulator-superconductor quantum mixer theory," J. Appl. Phys., vol. 53, pp. 3806-3812, May, 1982.

[11] L. R. D'Addario, "Saturation of the SIS mixer by out-ofband signals," IEEE Trans. Microwave Theory Tech., vol. 36, pp. 1103-1105, June 1988.

[12] M. J. Wengler, D. P. Woody, R. E. Miller and T. G Phillips, "A low noise receiver for millimeter and submillimeter wavelengths," Int. J. of Infrared and Millimeter Waves, vol. 6, pp. 697-706, 1985.

[13] G. J. Dolan, "Offset masks for lift-off photoprocessing," Appl. Phys. Lett., vol. 31, pp.337-339, Sept. 1977.

[14] L. N. Dunkleberger, "Stencil technique for the preparation of thin-film Josephson devices," J. Vac. Sci. Tech., vol. 15, pp. 88-90, Jan. 1978 .

[15] D. Rogovin and D. J. Scalapino, "Fluctuation phenomena in tunnel junctions," Ann. Phys., vol. 86, pp. 1-90, July 1974. 